\title{
Correction to: Primary antibody deficiencies in Turkey: molecular and clinical aspects
}

\author{
Sinem Firtina ${ }^{1,2}$ - Yuk Yin $\mathrm{Ng}^{3}$. Ozden H. $\mathrm{Ng}^{4}$. Ayca Kiykim ${ }^{5}$ - Esra Yucel Ozek ${ }^{6}$ - Manolya Kara ${ }^{7}$. Elif Aydiner ${ }^{8}$. \\ Serdar Nepesov ${ }^{9}$. Yildiz Camcioglu ${ }^{5}$. Esra H. Sayar ${ }^{10}$. Ezgi Yalcin Gungoren ${ }^{8}$. Ismail Reisli ${ }^{10}$. Selda H. Torun $^{6}$. \\ Sule Haskologlu ${ }^{11} \cdot$ Tuba Cogurlu $^{12} \cdot$ Aysenur Kaya $^{7} \cdot$ Sukru Cekic $^{13} \cdot$ Safa Baris $^{8} \cdot$ Ugur Ozbek $^{14} \cdot$ Ahmet Ozen $^{8}$. \\ Muge Sayitoglu ${ }^{1,15}$
}

Published online: 19 October 2021

(c) Springer Science+Business Media, LLC, part of Springer Nature 2021

\section{Correction to: Immunologic Research https://doi.org/10.1007/s12026-021-09242-z}

The original published version of this article contained a mistake in one of the affiliations. The correct affiliation of author Manolya Kara (7) should read:

Istinye University Faculty of Medicine, VM MedicalPark Pendik Hospital, Pediatric Infectious Diseases Clinic, Istanbul, Turkey

The original article has been corrected.

The original article can be found online at https://doi.org/10.1007/ s12026-021-09242-z

Muge Sayitoglu

mugeay@istanbul.edu.tr

1 Istanbul University Graduate School of Health Sciences, Istanbul, Turkey

2 Department of Bioinformatics and Genetics, Engineering and Natural Sciences Faculty, Istinye University, Istanbul, Turkey

3 Department of Genetics and Bioengineering, Istanbul Bilgi University, Istanbul, Turkey

4 Department of Medical Biology, Acibadem School of Medicine, Acibadem Mehmet Ali Aydinlar University, Istanbul, Turkey

5 Department of Infectious Diseases and Clinical Immunology, Cerrahpasa School of Medicine, Istanbul University, Istanbul, Turkey

6 Department of Clinical Immunology, Istanbul School of Medicine, Istanbul University, Istanbul, Turkey

7 Istinye University Faculty of Medicine, VM MedicalPark Pendik Hospital, Pediatric Infectious Diseases Clinic, Istanbul, Turkey
Publisher's note Springer Nature remains neutral with regard to jurisdictional claims in published maps and institutional affiliations.
8 Department of Pediatric Allergy and Immunology, Marmara University, Istanbul, Turkey

9 Department of Pediatric Allergy Immunology, Medipol University, Istanbul, Turkey

10 Department of Pediatric Immunology and Allergy, Meram Medical Faculty, Necmettin Erbakan University, Konya, Turkey

11 Department of Pediatric Immunology and Allergy, Ankara University School of Medicine, Ankara, Turkey

12 Department of Pediatric Immunology and Allergy, Kocaeli University, Kocaeli, Turkey

13 Department of Pediatric Immunology, Uludag University School of Medicine, Bursa, Turkey

14 Department of Medical Genetics, Acibadem School of Medicine, Acibadem Mehmet Ali Aydinlar University, Istanbul, Turkey

15 Department of Genetics, Aziz Sancar Institute of Experimental Medical Research, Istanbul University, Istanbul, Turkey 